\title{
Atrial fibrillation ablation: Limitations of pulmonary vein ablation catheter technology
}

We read with interest the paper by Koźluk et al. [1], which brings several points about multi-electrode duty-cycled radiofrequency ablation:

- The outcome in patients with paroxysmal atrial fibrillation $(\mathrm{AF})$ was lower when compared to 1 - and 5-year follow-up after single cryoballoon ablation procedure performed with higher follow-up regime (7- and not 1-day Holter ECG) [2]. Most triggers originate in left atrium (LA)-pulmonary vein (PV) junction [3]. Pulmonary vein isolation (PVI)-focused ablation, which eliminates triggers, and partially eliminates substrate located near LA-PVs junction, results in high success rate in such patients. Failure of PVI in this group is rather a result of incomplete isolation of LA-PVs triggers or existing non-LA-PVs triggers. Therefore, it would be interesting to know the percentage and $\mathrm{PV}$ anatomy pattern of reconnections in these patients. Was AF recurrence more often observed in atypical PVs?

- Pulmonary vein ablation catheter (PVAC) [1] focuses on PVs only. Unfavorable results in patients with persistent $\mathrm{AF}$ are not surprising. $\mathrm{AF}$ has tendency to become more persistent over time [4]. The progression of electrical and structural remodeling of atria promotes both reentry and ectopic activity which can serve both as substrate and trigger for AF [4]. Sole $\mathrm{PVs}$ isolation has low effectiveness in such not-PV-trigger-dependent AF.
- Recently, safety precautions for PVAC-procedures have been reported [5], which makes trans-septal puncture with uninterrupted warfarin more demanding and the PVAC-procedure more dependent on operator's experience.

- Consequently, PVAC ablation, with a tantalizing "single-shot" approach, should be rather reserved for carefully selected population of patients with PV-trigger-dependent AF and performed by an experienced operator.

Conflict of interest: None declared

\section{References}

1. Kozluk E, Balsam P, Peller M et al. Efficacy of multi-electrode duty-cycled radiofrequency ablation in patients with paroxysmal and persistent atrial fibrillation. Cardiol J, 2013; 20: 618-625.

2. Neumann T, Wojcik M, Berkowitsch A et al. Cryoballoon ablation of paroxysmal atrial fibrillation: 5-year outcome after single procedure and predictors of success. Europace, 2013; 15: 1143-1149 .

3. Weerasooriya R, Khairy P, Litalien J et al. Catheter ablation for atrial fibrillation: Are results maintained at 5 years of follow-up? J Am Coll Cardiol, 2011; 57: 160-166.

4. Xu Y, Sharma D, Li G, Liu Y. Atrial remodeling: New pathophysiological mechanism of atrial fibrillation. Med Hypotheses, 2013; 80: 53-56.

5. Verma A, Debruyne P, Nardi S et al. Evaluation and reduction of asymptomatic cerebral embolism in ablation of atrial fibrillation, but high prevalence of chronic silent infarction: Results of the evaluation of reduction of asymptomatic cerebral embolism trial. Circulation Arrhythmia Electrophysiol, 2013; 6: 835-842.

Maciej Wójcik, $M D^{1,2}$; Alexander Berkowitsch, $P h D^{2}$; Malte Kuniss, $M D^{2}$; Thomas Neumann, $M D^{2}$ ${ }^{1}$ Department of Cardiology, Medical University of Lublin, SPSK Nr 4, ul. Jaczewskiego 8, 20-954 Lublin, Poland, e-mail:m.wojcik@am.lublin.pl ${ }^{2}$ Department of Cardiology, Kerckhoff Heart and Thorax Center, Bad Nauheim, Germany 